\section{A Case of Drug-Induced Colitis Complicating the Administration of Hydroxycarbamide}

Gastrointestinal toxicity associated with the use of anti-cancer drugs, especially 5fluorouracil (5-FU), is a common problem in the treatment of malignant disease [1, 2]. Hydroxycarbamide is a commonly used antimetabolite, which is the firstline anticancer drug used in the treatment of chronic myeloblastic leukemia (CML). No cases of hydroxycarbamide-induced colitis have so far been reported.

A 62-year-old man was admitted in order to receive chemotherapy for CML. A few days after hydroxycarbamide administration $(2000 \mathrm{mg} /$ day), the patient developed abdominal pain and severe diarrhea. Infectious colitis and antibiotic-associated colitis were excluded clinically. The abdominal pain and diarrhea gradually worsened, despite treatment. A colonoscopic examination carried out 2 weeks after the start of hydroxycarbamide treatment showed multiple irregularly demarcated ulcers, extending from the sigmoid colon to the cecum (Figure 1). Histological specimens showed inflamed colonic mucosa with degenerated and regenerative epithelial crypts, some of which were dilated, and revealed mild atypia, with many apoptotic bodies (Figure 2). In view of these characteristic histological and endoscopic features, a diagnosis of colitis induced by the anticancer drug was made. The hydroxycarbamide treatment was stopped, and total parenteral nutrition

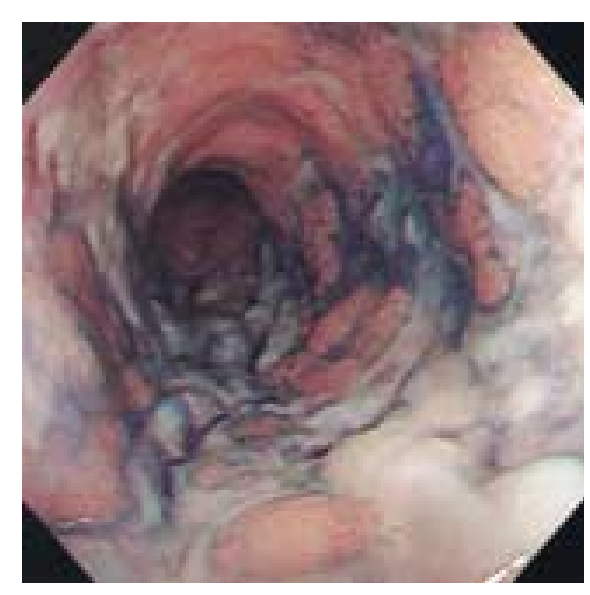

Figure 1 Colonoscopic view of irregularly demarcated ulcers in the descending colon was initiated. The diarrhea and abdominal pain rapidly improved. Two weeks later, colonoscopy demonstrated a marked improvement in the ulcers, in terms of number and size. Hematological remission was induced by interferon- $\alpha$ chemotherapy, and the patient has had no further abdominal symptoms for about a year.

Colitis induced by anticancer drugs is not a rare condition $[3,4]$, but its endoscopic features have only rarely been reported in the literature. In addition, there have been no previous reports of severe colitis induced by hydroxycarbamide, as in the present case. The endoscopic features in this case were similar to those of colitis induced by 5 -FU, described in a previous report [5]. In the present case, both the endoscopic examination and the histopathological findings in the biopsy specimen were very useful for the diagnosis of
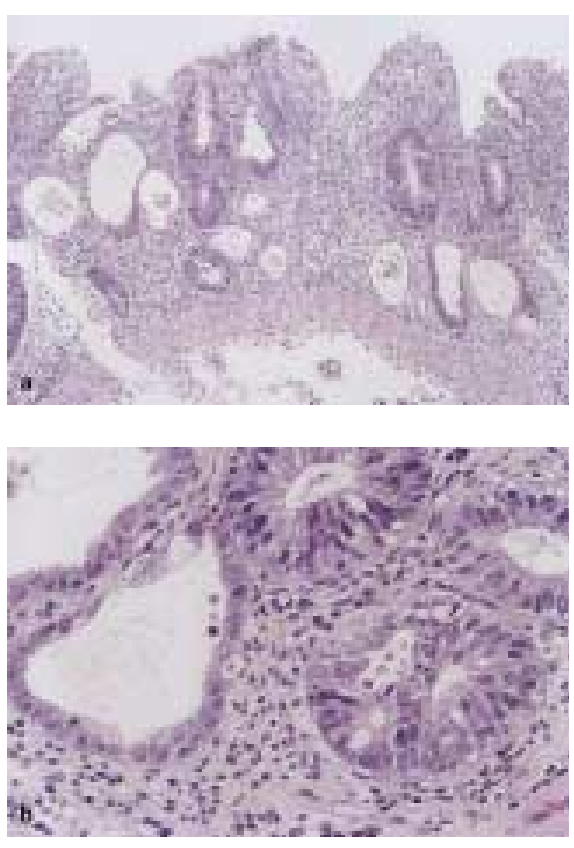

Figure 2 a Photomicrograph of the inflamed colonic mucosa, with degenerated and regenerative epithelial crypts (hematoxylin-eosin, original magnification $\times 25$ ) . b Some of the crypts were dilated and showed mild atypia, with many apoptotic bodies (hematoxylin-eosin, original magnification $\times 100$ ) drug-induced diarrhea and in reaching a treatment decision.

Y. Sadamoto ${ }^{1}$, T. Ueda ${ }^{1}$, M. Matsumoto ${ }^{1}$, M. Kubokawa ${ }^{1}$, K. Ito ${ }^{1}$, H. Kubo ${ }^{1}$, M. Tanaka ${ }^{1}$, N. Harada ${ }^{1}$, K. Muta ${ }^{1}$, H. Nawata ${ }^{1}$, M. Takata ${ }^{2}$, T. Yao $^{2}$

${ }^{1}$ Dept. of Medicine and Bioregulatory Science, Graduate School of Medical Sciences, Kyushu University, Fukuoka, Japan

${ }^{2}$ Dept. of Surgical Pathology, Graduate School of Medical Sciences, Kyushu University, Fukuoka, Japan

\section{References}

${ }^{1}$ Slavin RE, Dias MA, Saral R. Cytosine arabinoside-induced gastrointestinal toxic alterations in sequential chemotherapeutic protocols. Cancer 1978; 42: 1747 - 1759

2 Milles SS, Muggia AL, Spiro HM. Colonic histologic changes induced by 5-fluorouracil. Gastroenterology 1962; 43: $391-399$

${ }^{3}$ Cucmore MA, Silva J, Fekety R et al. Clostridium difficile colitis associated with cancer chemotherapy. Arch Intern Med 1982; 142: 333 - 335

${ }^{4}$ Iveson TJ, Chan A. Pseudomembranous colitis complicating chemotherapy. Lancet 1992; 339: 192 - 193

${ }^{5}$ Fukuda K, Kazami A, Ninomiya I et al. 5FU induced colitis: report of a case. Stomach Intest 2000; 35: 1185-1190

\section{Corresponding Author}

\section{Y. Sadamoto, M.D.}

Dept. of Medicine and Bioregulatory Science

Graduate School of Medical Sciences

Kyushu University

3-1-1 Maidashi, Higashi-ku

Fukuoka 812-8582

Japan

Fax: $\quad+81-92-642-5287$

E-mail: ysada@intmed3. med.kyushu-u.ac.jp 